\title{
LINGUISTIQUE
}

\author{
INGEBORGA BESZTERDA
}

Université Adam Mickiewicz, Poznań

\section{ALCUNE CONSIDERAZIONI INERENTI ALL'ARCHITETTURA DELL'ITALIANO CONTEMPORANEO}

\begin{abstract}
Beszterda Ingeborga, Alcune considerazioni inerenti all'architettura dell'italiano contemporaneo [Some remarks on the architecture of contemporary Italian]. Studia Romanica Posnaniensia, Adam Mickiewicz University Press, Poznań, vol. XXXIII : 2006, pp. 85-95. ISBN 83-2321643-6, ISSN 0137-2475.
\end{abstract}

This article describes certain fundamental issues relates to the current linguistic situation in Italy: the analysis of reciprocal relations between the national language and the Italian dialects (diglossia, bilinguism); the internal variants of Italian language depending on geographical location, social stratification, circumstances of the communication and the communication channels; the patterns of repertory proposed by famous linguists; some crucial problems concerning contemporary linguistic situation in Italy as: the importance of the diatopic variation; the recognition of the tension between Italian standard language derived from literary tradition and a new common form of the standard language; the recognition of the existence of a substandard variant - popular Italian.

La situazione linguistica d'Italia presenta una forte eterogeneità, da una parte esiste una lingua nazionale, l'italiano e dall'altra esistono dialetti che nonostante alcune opinioni negative sul loro indebolimento determinato dalla cosiddetta italianizzazione (Sobrero, $1978: 18$ ) conservano tuttavia una sorprendente vitalità. Il repertorio linguistico degli italiani si articola in un ventaglio di varietà, registri, sottocodici di diverso tipo (geografico, sociale, funzionale, situazionale), che vanno dalla variante letteraria più raffinata all'italiano fortemente regionalizzato o dialettizzato. Nel presente articolo si cercherà quindi di tracciare quella che, con un termine ripreso dalla linguistica germanofona, si chiamerebbe l'architettura dell'italiano, vale a dire, una sintesi del tipo delle varietà la cui somma costituisce la lingua contemporanea. 


\section{RETROSPETTIVA STORICA}

La situazione sociolinguistica italiana, all'epoca dell'unità (1861) presentava un forte squilibrio tra i poli linguistici fondamentali dell'italiano e del dialetto; lo squilibrio linguistico riproduceva quello sociale che esisteva tra le classi egemone e classi subalteme. L'italiano, la lingua nazionale ufficiale, è stato adoperato per secoli in cerchie ristrette di élites, particolarmente quelle di intellettuali e per occasioni solenni. D'altro canto, l'italiano era quasi esclusivamente riservato agli usi scritti, anche tra le classi dominanti, le quali per comunicare nella vita quotidiana, a seconda delle circostanze, si servivano sia di una grande lingua straniera di cultura (come il francese o lo spagnolo) sia del dialetto urbano locale. T. De Mauro (1983 : 43) considera che al momento dell'unificazione politica dell'Italia la percentuale degli italofoni si aggirava intorno al 2,5\%. Mentre A. Castellani (1982), in polemica con De Mauro, ritiene che gli italiani in grado di parlare la lingua nazionale erano all'epoca circa il 10\%. C. Grassi (2001 : 243) afferma che "alla proclamazione del Regno d'Italia sicuramente meno del $10 \%$ della popolazione conosceva la lingua nazionale". E noi siamo propensi a condividere questa opinione.

L'italiano si è sviluppato sullo scorcio del Duecento e Trecento come lingua scritta (all'inizio esclusivamente come lingua delle cancellerie, amministrazioni locali, mercanti) soprattutto negli ambienti della nuova classe emergente, quella di borghesia, mentre lo strumento di comunicazione orale era il dialetto. G. Sanga (1981 : 93) precisa : "Cette scission nette entre l'usage oral et l'usage écrit caractérise l'histoire linguistique italienne jusqu'en plein XIX siècle ». I dialetti, direttamente collegati ai vari volgari parlati nelle diverse regioni d'Italia, sono stati relegati per secoli ad un ambito d'uso familiare o a generi letterari minori, mentre la poesia e la prosa letteraria restavano ancorate all'italiano della tradizione trecentesca (le Tre Corone: Dante, Petrarca, Boccaccio). La prassi orale si estendeva quasi esclusivamente all'intemo del polo dialettale che era notevolmente stratificato a seconda delle classi sociali, e dall'altra parte enormemente frammentato in relazione ad aree geografiche. Questo stato di cose ha per lungo tempo contribuito a conservare nell'italiano una fondamentale scarsità lessicale connessa a particolari settori, quali l'artigianato, la gastronomia, la vita domestica, la flora, la fauna. L. Coveri (1998: 19) rileva: "Questa carenza viene a farsi sentire nel momento in cui l'italiano comincia non solo ad essere usato al posto del dialetto o del latino, ma serve a trattare argomenti affrontati fino a quel momento solo in dialetto (i mestieri, gli utensili e le attività della casa, gli animali, le piante) o in latino (i trattati di flora e di fauna)". Da questo fatto deriva inoltre, nell'italiano contemporaneo la particolare polimorfia lessicale che mantiene viva una moltitudine di termini regionali, diversi da zona a zona (geosinonimi) per designare vari oggetti domestici, oppure più termini regionali e uno più specifico, di solito di origine greca o latina, per indicare nomi di piante o di animali. Il fenomeno menzionato qui sopra, che si è lasciato notare soprattutto a partire dall'unità d'Italia, era favorito dal fatto che "lo 
sfasciamento dei dialetti, la loro adeguazione, soprattutto fonologica, all'italiano, ha reso sempre più facile trasferire parole e costrutti di origine dialettale nell'alveo della lingua comune" (De Mauro, op. cit. : 141).

\section{LE VARIETÀ DEL REPERTORIO}

Una condizione piuttosto complessa contraddistingue l'italiano come lingua in cui le varietà hanno spesso caratteristiche in comune e si intersecano in più punti. Nell'ambito del presente articolo si cercherà di proporre alcuni modelli del repertorio di varia complessità, con diverse classificazioni e parametri in relazione ad autori e ad una terminologia, non sempre univoca, da loro impiegata. Prima di addentrarci nei particolari dell'analisi in questione occorre, a titolo di introduzione, delineare il quadro generale delle dinamiche linguistiche in Italia.

Innanzitutto bisogna osservare che l'italiano pur essendo la lingua nazionale non costituisce l'unico strumento di comunicazione di tutti gli italiani. Di norma vengono ritenuti parlanti nativi dell'italiano tutti coloro che "hanno come lingua della socializzazione primaria l'italiano o un dialetto del gruppo italo-romanzo" (G. Berruto, $1999: 3$ ). Si accetta qui la posizione di Pellegrini (1977: 17) che delinea cinque "sistemi dell'italoromanzo" includendovi i domini italiano-toscano, settentrionale, centro-meridionale, friulano e sardo, aventi tutti "da tempo, come lingua guida (...) l'italiano". Data la loro distanza strutturale reciproca, in genere non molto diversa da quella che intercorre fra le varie lingue romanze maggiori e minori, i dialetti italiani vanno però considerati "varietà linguistiche a sé stanti, e non semplici varietà dell'italiano a coloritura locale" (Berruto, ibidem). Lo sottolinea anche A.M. Mioni (1979 : 102): "I dialetti italiani vanno considerati a tutti gli effetti lingue diverse e non varietà dell'italiano standard". Il repertorio linguistico della comunità parlante italofona consta, accanto alla lingua italiana, di una quindicina di altre varietà romanze, a cui occorre aggiungere, per completare il panorama delle "lingue indigene" d'Italia, le cinque lingue o varietà romanze e le sei lingue o varietà non romanze delle aree minoritarie di parlata alloglotta. E interessante rilevare inoltre che non esiste un unico repertorio linguistico panitaliano, valido per tutti gli italiani: i concreti repertori linguistici vanno sempre riferiti alle singole regioni ed aree. Tutti hanno peraltro in comune la presenza dell'italiano e delle sue varietà, che funge da tratto unficatore nella molteplicità dei repertori. Il repertorio linguistico italoromanzo medio comprende quindi essenzialmente varietà dell'italiano e varietà dei dialetti (cfr. C. Grassi, $2001: 161$ ).

\subsection{RAPPORTI TRA LA LINGUA NAZIONALE E I DIALETTI}

Prima di addentrarci nei particolari, conviene precisare la nozione della lingua e quella del dialetto. A questo proposito ci proponiamo di ricorrere alla definizione in merito proposta da Migliorini (1966:43): 
Una lingua gode di uno statuto socio-culturale e politico garantito da un ordinamento statale, possicde una codificazione riconosciuta e accettata all'interno e fuori dello Stato nazionale, incide sulla tradizione letteraria storicamente consolidata e affidata a istituzioni scolastiche, viene adottata come mezzo normale di comunicazione interregionale $\mathfrak{c}$ in ogni settore di attività. I dialetti sono invece impiegati in arce geograficamente circoscritte (villaggio, città, sub-regione), in ambiti limitati e prevalentemente nella varietà orale.

La relazione di status e funzionale che intercorre tra l'italiano e il dialetto viene definita da alcuni linguisti (Berruto, $2003: 28$ ) come quella di diglossia, cioè la distribuzione del repertorio fra una varietà alta, per gli usi scritti e formali, e una varietà bassa, per gli usi parlati informali. J. Trumper (1982:7) propone di affinare questa distinzione introducendo termini di macrodiglossia e microdiglossia per indicare la differenza tra aree (o anche classi sociali) dove il dialetto è assai più debole. Siccome però in Italia vengono impiegati nel parlato quotidiano sia la varietà bassa (il dialetto) che la varietà alta (l'italiano) e quindi l'assetto linguistico non coincide perfettamente con i requisiti della diglossia segnalati da Feruguson (1964 : 429), G. Berruto (1999: 5) suggerisce di definire il repertorio italoromanzo come "una situazione di bilinguismo endogeno a bassa distanza strutturale con dilalia". Mediante il termine bilinguismo a bassa distanza strutturale vengono designati dei casi in cui si denota l'uso e la compresenza di due diversi (dia)sistemi linguistici, la cui differenza strutturale (si tratta di varietà romanze dello stesso ceppo e sottoposte all'influsso livellatore della lingua standard) è inferiore a quella riscontrabile tra nei repertori bilingui classici. Tale bilinguismo è di natura interna al sistema linguistico (quindi endogeno) e non deriva da migrazioni o spostamenti di popolazioni più o meno recenti. G. Berruto (op. cit. : 6) mentre caratterizza la relazione di status e funzionale tra la varietà alta e quella bassa ricorre alla nozione dilalia "vale a dire con entrambe le varietà impiegate/impiegabili nella conversazione quotidiana e con uno spazio relativamente ampio di sovrapposizione".

C. Grassi (op. cit. : 254) pur accettando il termine diglossia (riferito però alla primà metà del XX secolo) propone di specificarlo mediante l'espressione più appropriata, quella di diglossia senza bilinguismo. Lo scienziato fa notare che "in realtà, tutti conoscevano il dialetto, ma solo pochi dominavano anche l'italiano, cioè erano bilingui". Lo conferma anche A.M. Mioni (1979 : 108). Nel secondo Novecento, in seguito ai processi che favoreggiarono l'uso della lingua nazionale (urbanesimo, alfabetizzazione, migrazioni interne, sviluppo dei mezzi di comunicazione di massa, ecc) in contrapposizione all'uso del dialetto e al passaggio a una società basata sull'industria, sul commercio, sulle comunicazioni, la situazione è radicalmente cambiata. C. Grassi (1999:280) sostiene quindi che si tratti di "diglossia con diversi gradi di bilinguismo e di bilinguismo con diglossia" facendo notare "lo spostamento graduale da una prevalente diglossia a un prevalente bilinguismo".

Secondo A.M. Mioni (ibidem) la situazione italiana media (nelle regioni a un livello medio di sviluppo) andrebbe definita come quella di diglossia con 
bilinguismo sociale, cioè "la maggioranza della popolazione conosce sia il dialetto che l'italiano (...) e condivide le norme che regolano la scelta di una varietà dell'italiano o del dialetto". Il linguista esaminando casi particolari del quadro linguistico italiano non esclude tuttavia dei casi di bilinguismo sociale senza diglossia quando la maggioranza dei parlanti conosce sia l'italiano che il dialetto ma, a causa della notevole percentuale di immigrati provenienti da altre regioni, i dialetti essendo cosi diversi non si possono integrare nell'uso di tutta la comunità. E interessante inoltre rilevare la presenza delle zone di monolinguismo standard che "era presente una volta in tutta l'area dei dialetti toscani, e tendeva a inglobare anche vaste aree di Lazio, Umbria e Marche; attualmente tende ad espandersi nelle classi medio-alte di tutta l'Italia" (Mioni, op. cit. : 110). In conclusione, Mioni (op. cit. : 104) constata: "dall'unificazione alla seconda guerra mondiale abbiamo un periodo di transizione da un monolinguismo dialettale quasi assoluto (con un numero limitato di parlanti bilingui) alla sinaazione attuale, la cui tendenza più avanzata è verso il monolinguismo italiano.

Da un'indagine DOXA condotta nel 1988 (U. Vignuzzi, $1988: 241$ ) risulta comunque che circa il $34 \%$ (di contro il $25 \%$ del 1974) degli intervistati dichiara di parlare italiano in famiglia, mentre il $23 \%$ (di contro il $29 \%$ del 1974) usa sempre il dialetto. I dati sopra riportati permettono di concludere "quanto [sia] salda la situazione di bilinguismo più o meno diglottico tra dialetto e lingua per larga parte della popolazione" (U. Vignuzzi, op. cit. : 244).

Bisogna menzionare che l'unità culturale e linguistica prima e politica poi, hanno comportato, e in qualche caso, imposto l'adozione della lingua come normale strumento di comunicazione orale accanto ai dialetti, o addirittura in loro sostituzione il che si ripercuote sulla natura e specificità degli scambi reciproci tra due sistemi linguistici in questione. Infatti, C. Grassi (op. cit. : 281) rileva due aspetti importanti di questi contatti: "... i dialetti hanno trovato nella lingua la fonte pressoché unica (...) dei loro mutamenti, la lingua, a sua volta, si è rinnovata anche grazie agli apporti dialettali".

M. Mioni (1979 : 106) analizzando l'influsso dei dialetti sull'italiano standard lo riconduce a tre modelli teorici:

1) meccanismi di interferenza

2) tendenze alla semplificazione

3) tendenze all'ipercorrettismo.

D'altra parte si assiste al processo inverso, quello della penetrazione dell'italiano nei dialetti. E importante notare a questo proposito che il processo di italianizzazione dei dialetti si manifesta perlopiù nel lessico e non tende a penetrare nel profondo delle strutture costitutive lasciando praticamente intatte la fonetica e la morfologia (cfr. Berruto, 1999 : 29). Nonostante i loro contatti o persino casi di compenetrazione, non si può parlare di "convergenza", cioè di tendenza dell'italiano e dei dialetti a congiungersi in un punto ideale del tempo futuro, perché l'italiano tende ad allontanarsi dai dialetti in seguito ad un processo di "rinormativizzazione" 
interna (cfr. Berruto 1989 : 108). Notiamo che mentre si accenna al contatto, non si intende contatto fra "tutta" la lingua e "tutto" il dialetto, solo fra le varietà della lingua più basse e le varietà di dialetto più alte. Così, Grassi $(1999$ : 307) rileva che "le varietà locali di dialetto e soprattutto l'italiano standard si evolvono senza sostanziali influssi reciproci perché il contatto diretto, e pertanto più intenso, avviene solo tra l'italiano colloquiale e più o meno fortemente regionalizzato (italiano popolare) e i livelli più elevati dei dialetti (dialetto urbano, koiné subregionale)". In altre parole, più che ad una convergenza il contatto fra lingua e dialetti sta portando ad una moltiplicazione delle varietà sia della lingua che dei dialetti, cioè dei casi di commutazione / alternanza di codice ed enunciazione mistilingue, cioè "l'uso alternato di varietà d'italiano e varietà di dialetto nel corso dello stesso atto comunicativo da parte dello stesso parlante, o addirittura all' interno della stessa battuta o frase" (Berruto, 1999 : 31). La rilevanza e la frequenza del fenomeno viene attestata in molte regioni, il che viene documentato da recenti studi (p.es. Berruto 1990, Sobrero 1992).

\subsection{VARIETÀ INTERNE DELL'ITALIANO}

Le fondamentali dimensioni della variazione sincronica della lingua sono costituite: dall'area geografica in cui viene impiegata la lingua (in particolare, dalla regione di provenienza dei parlanti e dalla loro distribuzione geografica) - variazione diatopica; dallo strato o gruppo sociale a cui appartengono i parlanti (o, più specificamente, dalla posizione che il parlante occupa nella stratificazione sociale) variazione diastratica; dalla situazione comunicativa nella quale si usa la lingua variazione diafasica; dal canale attraverso cui la lingua viene usata - variazione diamesica (cfr. Coveri, $1998: 10$ ). Le quattro dimensioni indicate qui sopra rappresentano degli assi di riferimento lungo i quali si possono ordinare le varietà compresenti nello spazio di variazione dell'italiano contemporaneo. Ciascun asse può essere considerato come un continuum che unisce due varietà contrapposte come poli estremi fra cui si collocano varietà intermedie. Lungo l'asse della dimensione diatopica, nella quale si collocano gli italiani regionali, i poli sono costituiti dall'italiano standard normativo e dall'italiano regionale fortemente dialettizzante, Lungo l'asse diastratico, si passa dall'italiano colto ricercato all'italiano popolare basso; lungo l'asse diafasico, dall'italiano formale aulico all'italiano informale trascurato; lungo l'asse diamesico, dall'italiano "scritto-scritto" all'italiano "parlato-parlato" (Nencioni, 1976).

Se la distinzione in queste quattro dimensioni fondamentali permette di tracciare lo schema della la cosiddetta architettura dell'italiano contemporaneo, non va dimenticato che nelle reali varietà d'uso della lingua spesso le diverse dimensioni si intersecano, e le relative varietà possono funzionare allo stesso tempo in conformità di più assi di variazione. Berruto $(1999: 10)$ specifica: 
così i gerghi (...) e certi linguaggi settoriali si definiscono contemporaneamente sull'asse diastratico, in quanto propri di certi gruppi sociali, e sull'asse diafasico, in quanto svolgenti una particolare funzione in date classi di situazioni comunicative; un italiano fortemente marcato in diatopia sarà per lo più una varietà sociale bassa; l'italiano popolare, varietà diastratica tipica di fasce sociali non istruite, sarà per $\mathrm{i}$ suoi parlanti anche una varietà diafasica, il registro delle occasioni più formali. Difficili da collocare con precisione, anche se appartengono fondamentalmente alla dimensione diafasica, sono poi le varietà di lingua legate a movimenti culturali, a mode, a costumi più o meno passeggeri, ecc., come le varie modalità d'uso via via battezzate giomalisticamente come sinistrese, politichese, giomalese, e cosi via.

Come vediamo, nella situazione italiana, non è praticamente possibile contraddistinguere tra la variazione diatopica e quella diastratica, e marcatezza diastratica comporta solitamente marcatezza diatopica. Si potrebbe avanzare un'ipotesi secondo la quale esista fra le dimensioni di variazione un'attinenza tale da farle funzionare l'una dentro l'altra: la diastratia dentro la diatopia, la diafasia dentro la diastratia, la diamesia dentro la diafasia. Secondo questa regolarità, un parlante nel periodo della socializzazione primaria impara una varietà sociale dell'italiano tipica della propria regione, entro la quale impara vari registri appropriati a diverse situazioni, entro cui impara la fondamentale dicotomia fra parlato e scritto.

\section{MODELLI DEL REPERTORIO}

In questa sezione ci proponiamo di passare in rassegna vari modelli del repertorio medio elaborati nel quadro delle recenti ricerche linguistiche italiane; si farà tuttavia astrazione dalla parte del repertorio relativa al polo dialettale che non ci interessa da vicino in questa sede. Nonostante la moltitudine di concezioni si riscontrano alcune costanti che vengono a costituire i nodi del dibattito: il rapporto fra l'italiano standard e una varietà comune (media e d'uso); il peso della differenziazione delle varietà sull'asse geografico; il riconoscimento dell'italiano popolare come substandard (anche se non sempre inserito negli stessi assi di variazione); la non identità di italiano regionale, italiano regionale popolare e italiano comune regionale. Per un quadro d'insieme, delle divergenze e delle convergenze tra $\mathrm{i}$ vari modelli si veda la tavola sinottica di Berruto (1999: 26).

Il primo tentativo di sistemazione delle principali varietà della lingua italiana contemporanea è stato intrapreso da Pellegrini (1960) che riconosceva quattro "registri espressivi" fondamentali nel repertorio verbale di un parlante italiano medio: dialetti, koinai dialettali, italiano regionale e italiano standard. Si osservi che con il termine italiano regionale si intende "l'ampia gamma di fenomeni compresa fra l'italiano della tradizione letteraria e il dialetto" (Berruto, 2003 : 13); si sottolinea inoltre che in Italia la prima fonte di diversificazione degli usi linguistici 
è quella legata alla distribuzione geografica, cioè alla dimensione diatopica. Ispirandosi alla suddetta quadripartizione sono state indicate numerose proposte di classificazione delle varietà d'italiano nell'ambito del repertorio linguistico nazionale.

Un approfondimento alla ripartizione fissata da Pellegrini (op. cit.) viene suggerito da A.M. Mioni (1975) che distingue tre varietà fondamentali: italiano aulico, italiano parlato formale e italiano colloquiale-informale. Correlando poi queste varietà con la stratificazione sociale Mioni presume che un borghese padroneggia tutte e tre le varietà, un piccolo borghese padroneggia solo la seconda $\mathrm{e}$ la terza "con qualche puntata sull'italiano aulico, spesso con effetti comici, dovuti ad ipercorrettismo o comunque a insicurczza linguistica" (op. cit. : 21) mentre un contadino solo la terza.

Nel (1983) Mioni introduce una terminologia diversa portando a quattro le varietà: standard formale, standard colloquiale-informale, it. regionale, it. popolare regionale. Questo modello tenta di rendere conto dell'imbricarsi delle gamme di variazione diatopica, diastratica e diafasica; sembra difficile però collocare uno standard colloquiale-informale che non sia contrassegnato da peculiarità regionali.

De Mauro (1980: 107-112) condensa precedenti proposte in una quadripartizione, che delinea una gerarchia costituita da it. scientifico, it. standard, italiano popolare unitario e italiano regionale colloquiale. Anche in questo quadro sono presenti tutte e tre le dimensioni di variazione. Berruto (2003:15) si dichiara scettico quanto alla proposta di Mioni rilevando che "uno schema siffatto (...) sottovaluta l'importanza della pronuncia, la cui considerazione deve giocoforza promuovere più in alto, in una gerarchia di differenziazione, la dimensione geografica". La mancanza di spazio ci impedisce purtroppo di prendere posizione riguardo al quesito relativo all'italiano popolare unitario la cui nozione ha suscitato molte polemiche. Ci limiteremo a segnalare che si tratta di una varietà caratterizzata (in quanto popolare) soprattutto dalla forte presenza di elementi regionali. Il carattere unitario di questa varietà (cioè per tratti non coincidenti con quelli della lingua standard) va ricondotto al fattore generale dell'oralità dell'uso ed ai processi di interferenza (con le atre varietà) e di semplificazione.

Sanga (1981 : 102-105) aumenta invece decisamente il numero delle varietà, proponendo un modello che consta di ben otto varietà fondamentali per la sezione italiana di un repertorio che intenda rappresentare in maniera esauriente la realtà sociolingistica odiema: it. anglicizzato, it. letterario (standard), it. regionale, it. colloquiale, it. burocratico, it. popolare (unitario), it. dialettale, italiano-daletto. Le varietà sono sistemate "d'un maximum d'unité à un maximum de diversité: chaque variété successive (...) implique les localismes de la variété précedente" (ivi, 105); ogni varietà è messa in relazione con la fascia sociale che le è propria o tipica. L'it. anglicizzato si distingue per la presenza di anglicismi e di termini del lessico europeo dotto, e viene usato dall'alta borghesia con contatti internazionali, dai managers, e in parte dai giornalisti. L'it. colloquiale costituisce la realizzazione orale corrente e informale dell'it. regionale, impiegato da vaste fasce sociali che 
vanno dalla borghesia ai ceti medi; it. burocratico proviene "des ministères, des tribunaux, des offices publics, des diverses branches de l'administration étatique. Il né de l'écrit, sur le modale du langage juridique" (ivi, 98), si basa su un ideale scritto artificioso. L'it. dialettale, fondamentalmente orale, è la realizzazione dell'it. popolare da parte dei soggetti fortemente dialettofoni; mentre l'italiano-dialetto sarebbe una "variété caractérisée par le passage continuel de l'expression italienne à l'espression dialectale, par la production continuelle d'énoncés en langue mixte", ed è anch'esso usato dalle classi popolari (ivi, 103). Lo schema di Sanga, pur essendo molto interessante, pone tuttavia alcuni problemi di interpretazione perché presenta una forte eterogeneità delle categorie: gli assi diatopico, diastratico e diafasico sono mescolati con caratteri intemi all'aspetto linguistico. Inoltre, per quanto riguarda singole varictà, quella denominata italiano-dialetto suscita qualche dubbio, perché conformemente a quanto si è detto nel 2.1 , non rappresenta una varietà, bensì commutazione di codice e simili fenomeni.

Si deve a Trumper (Trumper, Maddalon, $1982: 18-24$ ) l'innovazione di introdurre la distinzione fra uso scritto e uso parlato proponendo due repertori diversi per ciascuna delle due classi di usi sull'asse diamesico. Cosi, vengono considerati: it. standard, it. sub-standard, it. interferito sub-standard (per lo scritto); it. regionale formale, it. regionale trascurato fortemente interferito (per l'orale). Ogni varietà viene ulteriormente determinata secondo i parametri di variazione che vi confluiscono: l'it. regionale informale è definito allo stesso tempo in diatopia, diastratia e diafasia, l'it. regionale formale solo in diatopia e diastratia. Come vediamo, lo schema di Trumper presenta molte novità rispetto a quelli tracciati finora. Oltre alla distinzione in due sottorepertori, appare immediata l'assenza (comunque giustificata) dell'italiano standard in senso stretto del repertorio orale, che prende in considerazione un'eccezionale difficoltà di trovare parlanti, anche molto colti, la cui pronuncia non tradisca coloriture regionali più o meno marcate. Le varietà sono designate in termini di proprietà linguistiche secondo la quantità di elementi substandard contenuti e la maggiore o minore interferenza con il dialetto. L'idea di attribuire la qualità di standard esclusivamente allo scritto, comporta qualche difficoltà di paragone con il repertorio orale, in cui la dimensione principale risulta la formalità relativa.

Le categorie di usi scritti e parlati come criterio di riferimento importante vengono anche prese in considerazione da Sabatini (1985) che introduce accanto allo standard un'entità particolare, l'italiano dell'uso medio: "un tipo di lingua che si differenzia dallo standard ufficiale più che per i tratti propriamente regionali, soprattutto perché è decisamente ricettivo dei tratti generali del parlato" (ivi, 156). Esaminiamo ora la lista di Sabatini: it. standard, it. dell'uso medio, it. regionale delle classi istruite, it. regionale delle classi popolari (italiano popolare). Le prime due varietà sono entrambe nazionali, costituite di tratti panitaliani, e si distinguono in diafasia, essendo l'una tipica dell'uso formale e l'altra dell'uso "mediamente formale e informale" (1985: 176). Osserviamo che la separazione tra it. regionale 
e it. dell'uso medio comporta alcuni problemi. Sabatini stesso (ivi, 174) nota che "nulla toglie il fatto che l'uso orale di questa varietà [l'it. dell'uso medio parlato e scritto] si accompagni, anche nei parlanti colti, a tratti specifici di pronuncia regionale", anche se "talune fasce sociali e generazionali anche i tratti di pronuncia regionale si vanno attenuando di molto, fino a ridursi a generiche sfumature riferibili solo alle grandi aree geolinguistiche". Un altro problema deriva dalla natura stessa dell'it. dell'uso medio, che sembra "coprire un raggio forse eccessivamente ampio di variazione diafasica, se va dalle classi di impieghi non auliche a quelle del tutto non sorvegliate, e che pare semmai un nuovo standard, più che una varietà contrapposta allo standard" (Berruto, 2003 : 17).

Tutti questi schemi, e tanti altri ancora, che sono stati presentati nell'ultimo trentennio per rendere conto della composizione del repertorio italoromanzo medio, pur indicandone i nodi e le trame principali, non riescono a cogliere il quadro pieno della reale molteplicità degli usi riscontrabili nel comportamento linguistico degli italofoni. Nonostante la notevole eterogeneità terminologica, ed in alcuni casi, sovrapposizioni nelle etichette con cui vengono designate le varietà, si può osservare una certa intesa quanto ad alcuni problemi salienti dell'attuale situazione linguistica italiana:

il rilievo della differenziazione diatopica; il riconoscimento di una tensione fra l'italiano standard della tradizione letteraria e una nuova forma comune, media di uso della lingua standard in parte nuova, ma soprattutto ben più radicata nella comunità di quanto fosse nel passato; il riconoscimento dell'esistenza di una varietà sub-standard ben consolidata, l'italiano popolare (Berruto, $1999: 20$ ).

In conclusione si può affermare che la rassegna dei modelli di repertori di varietà dell'italiano sono stati proposti a livello nazionale, e vanno considerati validi per il repertorio medio di una qualsiasi regione italiana.

\section{BIBLIOGRAFIA}

Berruto G. (1989), Tra italiano e dialetto, in : G. Holtus, M. Metzeltin, M. Pfister (a c. di), La dialettologia italiana oggi. Studi offerti a Manlio Corelazzo. Narr, Tübingen, pp. 107-122.

Berruto G. (1990), Italiano regionale, commutazione del codice e enunciati mistilingui, in : M. Cortelazzo, A.M. Mioni (a c. di), L'italiano regionale. Bulzoni, Roma, pp. 105-128.

Berruto G. (1999), Le varietà del repertorio, in : A.A. Sobrero (a c. di), Introduzione all 'italiano contemporaneo. La vuriazione e gli usi. Laterza, Roma - Bari, pp. 3-36.

Berruto G. (2003), Sociolinguistica dell 'italiano contemporaneo. Carocci, Roma.

Castellani A. (1982), Quanti erano gli italofoni nel 1861? Studi Linguistici Italiani 8, 3-26.

Coveri L. (1978), Chi parla dialetto, a chi e quando in Italia? Un'inchicsta DOXA, La ricerca dialettale, 2, 331-341.

Coveri L., Benucci A., Diadori P. (a c. di) (1998), Le varietà dell'italiano. Bonacci Editore, Roma. 
De Ma u ro T. (1983), Storia linguistica dell'talia unita. Laterza, Roma - Bari.

Grassi C. (1999), Italiano e dialetti, in : A.A. Sobrero (a c. di), Introduzione all'italiano contemporaneo. La variazione e gli usi. Laterza, Roma - Bari, pp. 279-308.

Grassi C., Sobrero A.A., Telmon T. (2001), Fondamenti di dialettologia italiana. Laterza, RomaBari.

Migliorini B. (1966), Linguistica. Le Monnier, Firenze.

Mioni A.M. (1975), Per una sociolinguistica italiana. Note di un non sociologo, in : J.A. Fishman, La sociologia del linguaggio. Officina, Roma, pp. 7-56.

Mioni A.M. (1979), La situazione sociolinguistica italiana: lingua, dialetti, italiani regionali, in : A. Colombo (a c. di) Guida all'educazione linguistica. Fini, modelli, pratica didatica. Zanichelli, Bologna.

Mioni A.M. (1983), Sociolinguistica, in : AA.VV., Aspetti linguistici della comunicazione. La Garangola, Padova, pp. 135-149.

Nencioni G. (1976), Parlato-parlato, parlato-scritto, parlato-recitato, Strumenti critici, 10, 1-56.

Pellegrini G.B. (1960), Tra lingua e dialetto in Italia, Studi mediolatini e volgari, 8, 137-153.

Peruzzi E. (1967), Una lingua per gli italiani. Edizioni Rai, Torino.

Sabatini, F. (1985), L'italiano dell'uso medio: una realtà tra le varietà linguistiche italiane, in : G. Holtus, E. Radtke (a c. di), Gesprochenes Italienisch in Geschichte und Gegenwart. Narr, Tübingen, pp. 154-184.

Sabatini F. (1990), Italiani regionali e italiano dell'uso medio, in : M. Cortelazzo, A. Mioni (a c. di), L'italiano regionale. Bulzoni, Roma.

Sanga G. (1981), Les dynamiques linguistiques de la société italienne (186I-1980) : de la naissance de l'italien populaire à la diffusion des ethicismes linguistiques, Langages, 61, 93-109.

Sobrero A.A. (1978), L'italiano impopolare, in : I padroni della lingua. Guida Editori, Napoli.

Sobrero A.A. (a c. di) (1992), Il dialetto nella conversazione. Ricerche di dialettologia pragmatica. Congedo, Galatina.

Trumper J. (1977), Ricostruzione nell Italia settentrionale: sistemi consonantici, in : AA.VV. Problemi della ricostruzione in linguistica. Bulzoni, Roma, pp. 259-310.

Trumper J., MaddaIon M. (1982), L'italiano regionale tra lingua e dialetto. Edizioni Brenner, Cosenza.

Vignuzzi U. (1988), Chi parla ancora in dialetto?, Italiano e Oltre, 5, 241-245. 